\title{
Depolarization of elliptically polarized light in birefringent crystal
}

\author{
Ł. Michalik, M. Redek, P. Makowski, A.W. Domański* \\ Faculty of Physics, Warsaw University of Technology, Koszykowa 75, 00-662 Warszawa
}

Received December 17, 2009; accepted December 30, 2009; published December 31, 2009

\begin{abstract}
During light propagation in birefringent medium, the state of polarization (SOP) changes and in case of partially coherent light the degree of polarization (DOP) also changes. Depolarization of linearly and circularly polarized light in birefringent media has been investigated experimentally for several years. Modified Mueller-Stokes formalism with the help of the depolarization matrix has allowed predictions of all the results of such research. However, the linear and the circular state of polarization are just the special cases providing the highest symmetry. By so far, the modified Mueller-Stokes method has not been verified in the case of elliptically polarized light.

In the paper we present the results of research on depolarization of elliptically polarized light passing through a single birefringent crystal. The performed measurements verify quantitatively the theoretically predicted dependence of the output DOP and the ellipticity and azimuth angles of the polarization state of the light coupled into the crystal
\end{abstract}

The state of polarization (SOP) of partially coherent light is described by the Born-Wolf formalism, by means of the coherency matrix [1]:

$$
[\mathbf{K}]=\left[\begin{array}{cc}
J_{x x} & J_{x y} \\
J_{y x} & J_{y y}
\end{array}\right]=\left[\begin{array}{cc}
\left\langle E_{x} E_{x}^{*}\right\rangle & \left\langle E_{x} E_{y}^{*}\right\rangle \\
\left\langle E_{y} E_{x}^{*}\right\rangle & \left\langle E_{y} E_{y}^{*}\right\rangle
\end{array}\right],
$$

where \langle\rangle stands for temporal mean value, ${ }^{*}$ means the complex conjugate value, and $\mathrm{E}_{\mathrm{x}}$ and $\mathrm{E}_{\mathrm{y}}$ are electric field components of the lightwave.

One important remark is that we can separate out the part of the totally polarized light from the entire intensity of the incident beam and that is why we can rewrite the coherency matrix as a sum of two matrices [1]. A consequence of this property is that we get an expression for degree of polarization (DOP) in the form:

$$
D O P=\frac{\mathrm{I}_{\mathrm{pol}}}{\mathrm{I}_{\text {tot }}}=\sqrt{1-\frac{4 \mathrm{det}[\mathbf{K}]}{\left(\mathrm{J}_{\mathrm{xx}}+\mathrm{J}_{\mathrm{yy}}\right)^{2}}} \leq 1 .
$$

The dependence of the intensity of electric field vector on the angle $\Theta$ between $\mathrm{X}$ axis and vector $\mathbf{E}$ is as follows:

*E-mail: domanski@if.pw.edu.pl

$$
\begin{gathered}
\mathrm{I}(\Theta, \Delta \delta)=\sqrt{\frac{\varepsilon_{0}}{\mu_{0}}}\left\langle E(t, \Theta, \Delta \delta) E^{*}(t, \Theta, \Delta \delta)\right\rangle= \\
=J_{x x} \cos ^{2} \Theta+J_{y y} \sin ^{2} \Theta+ \\
+2 \sqrt{J_{x x}} \sqrt{J_{y y}} \sin \Theta \cos \Theta\left|\mu_{x y}\right| \cos \left(\beta_{x y}-\Delta \delta\right), \\
\mu_{x y}=\left|\mu_{x y}\right| \exp \left(i \beta_{x y}\right)=\frac{J_{x y}}{\sqrt{J_{x x}} \sqrt{J_{y y}}},
\end{gathered}
$$

where $\mu_{x y}$ is the complex degree of coherence. Equation (3) is formally similar to the expression describing interference of two wave packets in a Michelson interferometer [1]. There we have $\gamma(\tau)$ which is also called the complex degree of coherence and it is responsible for the contrast of the interference pattern decreasing with increasing delay between two wave packets.

Born and Wolf assumed that $\left|\mu_{x y}\right|=|\gamma(\tau)|$. It means that interference between two parallel waves is analogous to the superposition of two orthogonally polarized modes, whose relative phase shift is increasing in the birefringent medium. Because of that shift, a smaller and smaller part of light is polarized.

After simple calculations on expression (2) we can rewrite it as $[2,3]$ :

$$
D O P=\sqrt{1-\frac{4\left(1-|\gamma(\tau)|^{2}\right)}{\left(\frac{\mathrm{E}_{0 \mathrm{x}}}{\mathrm{E}_{0 \mathrm{y}}}+\frac{\mathrm{E}_{0 \mathrm{y}}}{\mathrm{E}_{0 \mathrm{x}}}\right)^{2}}}=\sqrt{1-\frac{4\left[1-\exp \left(-2 \eta_{S}\right)\right]}{\left(\frac{\mathrm{E}_{0 \mathrm{x}}}{\mathrm{E}_{0 \mathrm{y}}}+\frac{\mathrm{E}_{0 \mathrm{y}}}{\mathrm{E}_{0 \mathrm{x}}}\right)^{2}}}
$$

where $\eta_{s}$ depends on the type of laser spectrum Lorentzian or Gaussian [2]:

$$
\eta_{S L}=\frac{|\Delta n| L}{\Delta L_{\text {Lor }}} \quad, \quad \eta_{S G}=\left(\frac{|\Delta n| L}{\Delta L_{\text {Gauss }}}\right)^{2} .
$$


Application of the coherency matrix to describe changes of the state of polarization after light propagation through an optical medium is inconvenient. Mathematical difficulties were solved by introducing the MuellerStokes matrix formalism, based on Stokes vectors [1]:

$$
[\mathbf{S}]=\left[\begin{array}{c}
\mathrm{S}_{0} \\
\mathrm{~S}_{1} \\
\mathrm{~S}_{2} \\
\mathrm{~S}_{3}
\end{array}\right]=\left[\begin{array}{c}
\mathrm{J}_{\mathrm{xx}}+\mathrm{J}_{\mathrm{yy}} \\
\mathrm{J}_{\mathrm{xx}}-\mathrm{J}_{\mathrm{yy}} \\
\mathrm{J}_{\mathrm{xy}}+\mathrm{J}_{\mathrm{yx}} \\
i\left(\mathrm{~J}_{\mathrm{yx}}-\mathrm{J}_{\mathrm{xy}}\right)
\end{array}\right]
$$

To include the depolarization phenomenon in the matrix formalism, the depolarization matrix $\mathbf{D}$ was introduced [2]:

$$
[\mathbf{D}]=\left[\begin{array}{cccc}
1 & 0 & 0 & 0 \\
0 & P_{C} & 0 & 0 \\
0 & 0 & P_{C} & 0 \\
0 & 0 & 0 & P_{C}
\end{array}\right]
$$

The state and degree of light polarization at the output of optical element is described as:

$$
\left[\mathbf{S}^{\text {out }}\right]=[\mathbf{D}][\mathbf{M}]\left[\mathbf{S}^{\text {in }}\right]
$$

where $\mathbf{M}$ is the Mueller matrix of the optical element. We can rewrite (5) using elements of the Stokes vector:

$$
D O P=P_{C}=\sqrt{1-\left[1-\exp \left(-2 \eta_{S}\right)\right]\left[1-\left(\frac{S_{1}^{\text {in }}}{S_{0}^{i n}}\right)^{2}\right]}
$$

Such a description is sufficient in the case of linearly polarized light incoming to the crystal. In our previous paper [4] we checked the depolarization of circular polarized light passing through the crystal. In this paper we report additional experiments with elliptically polarized light coupled into the crystal.

In Fig. 1 the experimental setup is presented.

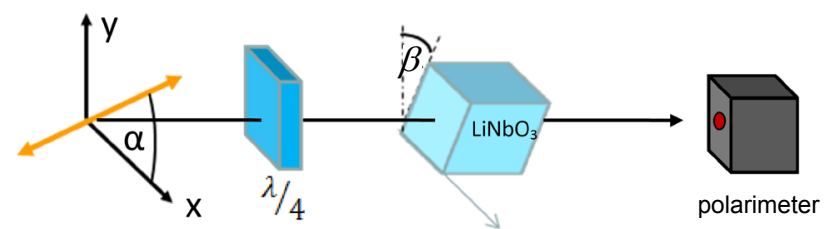

Fig.1 Experimental setup for measurement of the dependence between the ellipticity of the input beam and the DOP of the beam outgoing from the birefringent crystal
In this configuration the ellipticity of the light is changed by rotation of the linearly polarized light before the quarter-wave-plate $(\alpha)$. The rotation angle of the birefringent crystal $(\beta)$ changes the power distribution between axes.

A general state of polarization at the crystal input (X-Y coordinates) and at polarimeter (output) can be written as:

$$
\begin{gathered}
{\left[\mathbf{S}^{\text {in }}\right]=\left[\mathbf{M}_{N / 4}\right]\left[\mathbf{S}^{\text {linear }}(\alpha),\right.} \\
{\left[\mathbf{S}^{\text {out }}\right]=[\mathbf{D}]\left[\mathbf{M}(\beta)_{L_{\text {LiNbO }}}\right]\left[\mathbf{S}^{\text {in }},\right.}
\end{gathered}
$$

where the Mueller matrices of the quarter-wave-plate and the rotated crystal are respectively [5]:

$\left[\mathbf{M}_{\lambda / 4}\right]=\left[\begin{array}{cccc}1 & 0 & 0 & 0 \\ 0 & 1 & 0 & 0 \\ 0 & 0 & 0 & 1 \\ 0 & 0 & -1 & 0\end{array}\right]$

$\left[\mathbf{M}(\beta)_{L i N b O 3}\right]=$

$\left[\begin{array}{cccc}1 & 0 & 0 & 0 \\ 0 & \cos ^{2}(2 \beta)+\sin ^{2}(2 \beta) \cos (\delta) & (1-\cos (\delta)) \sin (2 \beta) \cos (2 \beta) & \sin (2 \beta) \sin (\delta) \\ 0 & (1-\cos (\delta)) \sin (2 \beta) \cos (2 \beta) & \sin ^{2}(2 \beta)+\cos ^{2}(2 \beta) \cos (\delta) & \cos (2 \beta) \sin (\delta) \\ 0 & \sin (2 \beta) \sin (\delta) & -\cos (2 \beta) \sin (\delta) & \cos (\delta)\end{array}\right]$,

and where

$\delta=2 \pi \Delta n l / \lambda$.

The parameters of the beam coupled into the crystal are as follows:

$$
\vartheta(\alpha)=\beta+\frac{1}{2} \cdot \arctan \left(\frac{S_{2}^{i n}}{S_{1}^{i n}}\right)=\beta,
$$

for the azimuth of the polarization ellipse and

$$
|\varphi(\alpha)|=\frac{1}{2} \cdot\left|\arcsin \left(\frac{S_{3}^{i n}}{\sqrt{\left(S_{1}^{\text {in }}\right)^{2}+\left(S_{2}^{i n}\right)^{2}+\left(S_{3}^{\text {in }}\right)^{2}}}\right)\right|=\alpha,
$$

for the ellipticity angle (arc tangent of ellipticity - ratio of elliptical semiaxes).

As a first step in our experiments we measured the polarization of light outgoing from the lithium niobate crystal at the angle $\beta=45^{\circ}$ (Fig.1). The degree of polarization was constant. Experimental points fit the theoretical line (calculated from equation (10) for a Lorentzian spectrum) of light source with a coherence length $\Delta L=2 \mathrm{~mm}$. We find confirmation of the fact that when the power distribution between birefringent axes is equal, $D O P$ is constant irrespective of ellipticity (Fig.2). 


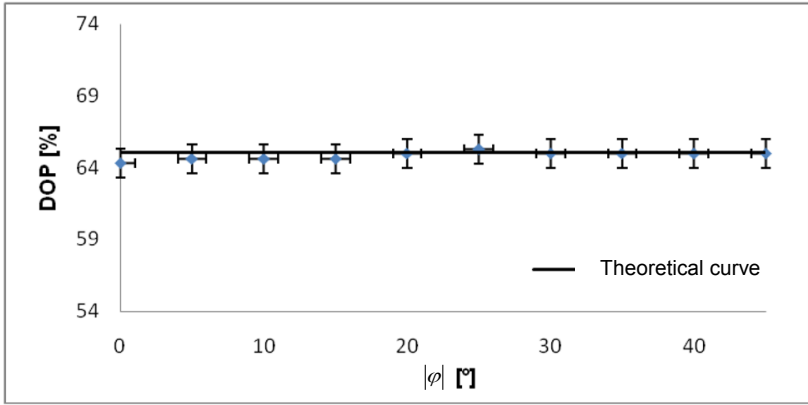

Fig.2 Dependence between DOP of output beam from the crystal and ellipticity angle of the input beam for an equal power distribution case

However ellipticity should be taken into consideration if the crystal angle is different than $\beta=45^{\circ}$ and the power distribution is not equal. In these cases $D O P(10)$ reaches it's minimum for ellipticity angle $\varphi=45^{\circ}$ (circular SOP). For $\varphi=0^{\circ}$ we have the maximum DOP. For example when $\beta=15^{\circ}$ or $\beta=30^{\circ}, D O P$ decreases as we can see in Fig.3.

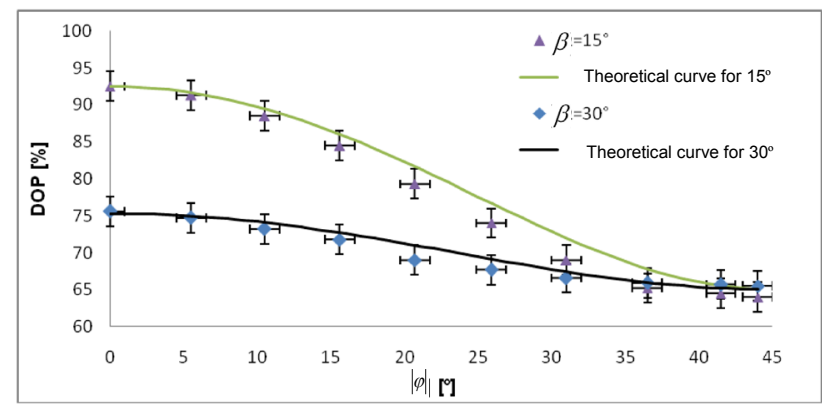

Fig.3 Dependence between DOP of output beam from the crystal and ellipticity angle of input beam for two arbitrary chosen angles of the crystal orientation

For the next measurements the input beam azimuth was aligned with the birefringent axis of the crystal. For $\varphi=0^{\circ}$, the input beam is linearly polarized and depolarization does not occur. For increasing ellipticity angle of the beam, the $D O P$ decreases in the same way as in the case of rotation of the linear polarization azimuth. In addition we used two light sources with different coherency length of emitted light. The depolarization of the light is stronger for the light source with the smaller coherence length $\Delta L$ (Fig.4).

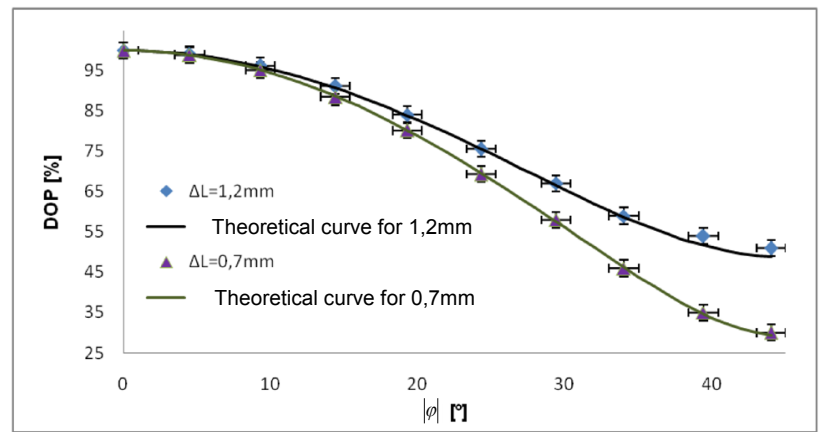

Fig.4 Dependence between the DOP of output beam from the crystal and the ellipticity angle of the input beam for the case when the azimuth of the polarization ellipse is aligned with the birefringent axis of the crystal

The experiment was carried out under the following conditions (Tab.1).

Tab.1 Experimental conditions
\begin{tabular}{|c|c|}
\hline laser spectrum & Lorentzian \\
\hline wavelength & $661 \mathrm{~nm}$ \\
\hline length of coherence & various \\
\hline crystal length & $10 \mathrm{~mm}$ \\
\hline crystal birefringence & 0,086 \\
\hline
\end{tabular}

The conclusion from these experiments is that the modified Mueller-Stokes matrix formalism is a very good method for describing depolarization phenomena, not only for linearly and circular polarized light but also for more a general case like elliptically polarized light. Calculations based on the Mueller-Stokes matrix formalism using the depolarization matrix gave accurate predictions for experiments not only for different birefringent crystals and polarization parameters, but also for different coherence lengths of the incoming light.

\section{References}

[1] M. Born, E. Wolf "Principles of Optics", Cambrige University Press, 1997

[2] A.W. Domanski, "Polarization degree fading during propagation of partially coherent light through retarders", Opto-Electronics Review 13(2), 171-176, 2005

[3] A.W. Domanski, D. Budaszewski, M. Sierakowski, Tomasz R. Woliński "Depolarization of partially coherent light in liquid crystals" Opto-Electronics Review., 14, no 4, pp. 61-66, 2006

[4] A. W. Domanski, M. Redek, D. Budaszewski „Depolarization of circularly polarized light in birefringent crystal", Photonics Letters of Poland, Vol 1, No 2, 67-69, 2009

[5] D. Goldstein "Polarized Light", Marcel Dekker Inc., 2003 